01,11

\title{
Оценка влияния икосаэдрических „магических“ чисел на термическую стабильность малых нанокластеров серебра
}

\author{
(ㄱ Д.А. Рыжкова, С.Л. Гафрнер, Ю.Я. Гафрнер \\ Хакасский государственный университет им. Н.Ф. Катанова, \\ Абакан, Россия \\ ฯ E-mail: ygafner@khsu.ru \\ Поступила в Редакцию 22 ноября 2021 г. \\ В окончательной редакции 22 ноября 2021 г. \\ Принята к публикации 25 ноября 2021 г.
}

Методом молекулярной динамики с использованием модифицированного потенциала сильной связи TB-SMA проведен сравнительный анализ термически индуцированных структурных переходов в нанокластеpax серебра, числа атомов которых соответствовали „магическим“ числам икосаэдрической (Ih) структуры при вариации их начальной морфологии. Показано, что в случае исходной ГЦК-фазы формирование Ih-модификации, в зависимости от размера частицы, происходило либо на этапе предварительной термической релаксации, либо в ходе дальнейшего нагрева. При начальной аморфной морфологии характер структурных переходов претерпевал значительные изменения. Так, даже в случае кластеров $\mathrm{Ag}_{55}$, икосаэдрическое строение образовывалась только в 50-60\% проведенных опытов. На основе полученных данных сделан вывод, что для создания устойчивой Ih-структуры необходимо использовать процедуру термоциклирования.

Ключевые слова: нанокластеры, серебро, компьютерное моделирование, „магические“ числа, структура нанокластеров.

DOI: 10.21883/FTT.2022.03.52091.245

\section{1. Введение}

Наночастицы серебра (АgНЧ) приобретают сейчас все большую популярность в самых различных технологических сферах, таких как электроника, фотоника и медицина [1]. Рассмотрим только некоторые причины повышенного внимания к AgНЧ. Так широко используемые в современной жизни элементы солнечных панелей, сенсорные экраны, ЖК-дисплеи, органические электролюминесцентные панели, светодиоды (LED и OLED), часто применяют в своей конструкции электроды, изготовленные из напыленных пленок оксида индия и олова (ITO) [2,3]. Однако данные пленки имеют ряд серьезных недостатков, таких как высокая стоимость, хрупкость [4] и токсичный состав. Для решения этих проблем в настоящее время в качестве альтернативы рассматриваются иные материалы, такие как графен, углеродные нанотрубки или проводящие полимеры с добавлением металлических наноструктур [5-7]. Но только пленки из серебряных наночастиц превосходят ITО-пленки по коэффициенту пропускания света и электропроводности [8]. В этой связи отметим, что оптические свойства $\mathrm{AgHЧ} \mathrm{во} \mathrm{многих} \mathrm{случаях} \mathrm{определяются} \mathrm{локализованным}$ поверхностным плазмонным резонансом (LSPR), который сильно зависит от формы, размера НЧ и материала окружающей среды [9].

Другим интересным физическим явлением, использующим плазмонный эффект, стала поверхностно-усиленная рамановская спектроскопия (surface-enhanced Raman Scattering - SERS), обладающая чрезвычайной чувствительностью и высокой селективностью к исследуемому веществу, что имеет большое значение для химического анализа и изучения строения материи. Среди различных плазмонных материалов именно серебряные SERSподложки обеспечивают наиболее сильное рамановское усиление и поэтому наиболее перспективны для широкого практического применения [10].

Тип металла, размер, форма и степень агрегации частиц определяют энергетический диапазон (частоту) света, который может возбуждать плазмоны (теория рассеяния Ми). Поверхностный плазмонный резонанс можно контролировать, управляя формой AgHЧ, которая, в свою очередь, регулирует колебания электронов. Например, основная длина волны поглощения сферических AgНЧ (размер $10 \mathrm{~nm}$ ) составляет $\approx 400 \mathrm{~nm}$. С другой стороны, более крупные $\mathrm{AgHЧ} \mathrm{и} \mathrm{AgНЧ} \mathrm{различной}$ формы из-за иных режимов поглощения поглощают свет с другой длиной волны [11]. Так наночастицы серебра со средним размером $34 \pm 14 \mathrm{~nm}$ в виде треугольных призм вызывали свечение в синем диапазоне электромагнитных волн, a AgHЧ квазисферической формы со средним размером $16 \pm 7 \mathrm{~nm}$ обладали ярко выраженным желтым цветом [12].

Использование AgHЧ в биомедицине составляет более трети их общего применения и здесь также было отмечено влияние формы и внутреннего строения на свойства AgНЧ. При изучении бактерицидной активности сферических AgHЧ различного размера против E-coli, Bacillus subtilis и Staphylococcus aureus пришли к выводу, что их эффективность возрастает с уменьшением размера независимо от бактериальных штаммов [11], что может быть связано с более высокими площадями 
поверхности, которые способствуют прикреплению к клеточным мембранам. Сдругой стороны, исследования показывают, что реакционная способность и антибактериальная активность АgНЧ сильнее в частицах с преобладающими гранями $\{111\}$ с более высокой атомной плотностью по сравнению с другими морфологическими формами. Так была проведена проверка эффективности сферических и нитеобразных AgHЧ в отношении их антибактериальной активности к разным бактериям и было показано, что уровень смертности Klebsiella pneumoniae был выше при воздействии сферических $\mathrm{AgHЧ} \mathrm{с} \mathrm{более}$ крупными $\{111\}$ гранями по сравнению с АgНЧ в форме стержней [12]. Таким образом было обнаружено, что именно фасеты $\{111\}$ в большей степени ответственны за антибактериальную активность $\mathrm{AgHЧ,} \mathrm{что} \mathrm{ставит}$ вопрос о создании AgHЧ со сферическим (квазисферическим), декаэдрическим или октоэдрическим строением стабильным в области комнатных температур.

Следовательно, с точки зрения использования нанокластеров серебра в плазмонных и иных приложениях, представляется очень важным определение границ термической и размерной стабильности кристаллического строения и формы Ag-наночастиц. Для этого в работе будут рассмотрены возможные конфигурационные изменения кластеров серебра в процессе нагрева до температуры плавления и предпринята попытка обозначить размерные границы наночастиц, в которых может происходить структурный переход.

\section{2. Компьютерная модель}

Хорошо известно, что именно структура и взаимное расположение атомов во многом определяют свойства наночастиц. Помочь в прогнозировании их строения может описание механизмов образования и динамики изменения внутренней морфологии. Изучение этих процессов экспериментальными методами сопряжено с рядом существенных трудностей, обусловленных малыми размерами наночастиц, и поэтому более эффективными в данном случае могут оказаться методы компьютерного моделирования. На наш взгляд, именно такой метод является наиболее перспективным при исследовании структурных превращений, механизмов образования и роста наночастиц, определении равновесных конфигураций, термодинамических свойств и кинетики проходящих процессов, динамики их изменения под влиянием внешних условий: температуры, давления и т.д.

В представленной работе компьютерное моделирование нанокластеров серебра проводилось методом молекулярной динамики с использованием модифицированного потенциала сильной связи TB-SMA [13] который основан на предположении, что большая группа свойств переходных металлов может быть полностью определена из плотности состояний внешних $d$-электронов. Именно такой метод, в котором ион-ионное взаимодействие описано с учетом зонного характера связи и ко- роткодействующего парного потенциала отталкивания, на наш взгляд, способен достаточно правильно описать некоторые характерные особенности малых металлических наносистем. Поэтому моделирование наночастиц серебра было проведено с использованием именно такого потенциала межатомного взаимодействия.

Компьютерный анализ всех протекающих в Ag-кластерах процессов проводился в каноническом NVT-ансамбле. Температура системы определялась посредством средней кинетической энергии атомов, рассчитанной при помощи скоростного алгоритма Верле с шагом по времени $1 \mathrm{fs}$. Начальные сферические кластеры были получены путем вырезания из идеальной ГЦК-решетки с последующей термической релаксацией при $20 \mathrm{~K}$. На этом этапе был сформирован первый ансамбль исследуемых частиц. Затем AgHЧ были ступенчато нагреты с целью полного разрушения дальнего порядка в них и резко охлаждены с высоким темпом отвода тепловой энергии до температуры $20 \mathrm{~K}$. Из полученного массива наночастиц были выделены те, что имели аморфное строение. Таким образом был сформирован второй ансамбль AgHЧ. Далее АgНЧ различной начальной морфологии ступенчато нагревались до температуры $1000 \mathrm{~K}$ с шагом $20 \mathrm{~K}$, а в области структурных переходов в шагом $5 \mathrm{~K}$. При каждом фиксированном значении частицы выдерживались в течение 1 ns.

Моделирование проводилось с использованием компьютерной программы MDNTP, разработанной Dr. Ralf Meyer, University Duisburg Germany. Наличие структурных переходов определялось по зависимости потенциальной энергии нанокластеров от температуры, программе- визуализатору OVITO и функции радиального распределения.

\section{3. Результаты и обсуждения}

Исходя из того, что вариации структурных свойств наноструктур серебра наиболее хорошо проявляются именно у кластеров малого размера, при проведении компьютерного анализа термической стабильности строения AgHЧ были выбраны частицы диаметром до $2.0 \mathrm{~nm}$. На наш взгляд причина подобного явления состоит в том, что при таких малых диаметрах AgHЧ по своим физико-химическим свойствам близки именно к молекулярным, а не объемным структурам, поскольку их линейные величины сопоставимы с длиной волны Ферми электронов ( $0.5 \mathrm{~nm}$ для $\mathrm{Ag})$ из-за чего здесь начинают превалировать квантовые эффекты. Другим важным следствием квантовых особенностей является то, что в наночастицах малого размера существенную роль в стабильности строения могут играть так называемые „магические“ числа различной природы.

В наших более ранних работах по данной тематике $[14,15]$ уже проводился анализ термической устойчивости структуры нанокластеров серебра диаметром до $2.0 \mathrm{~nm}$ при условии их начального ГЦК-строения. 
Было показано, что такой вид Ag-кластеров оказывается термически стабильным для наночастиц диаметром $D>1.8 \mathrm{~nm}(170-180$ атомов). Однако при меньших размерах сценарии термической эволюции внутреннего строения нанокластеров серебра значительно усложнялись, и в некоторых случаях наблюдалось образование y AgHЧ максимально возможной сферической формы, часто обладающей икосаэдрическим или декаэдрическим расположением атомов. Октоэдрическая огранка у наночастиц серебра таких размеров не фиксировалось даже в ходе нагрева до высоких температур.

Данный результат не вызывает удивления, так как в случае кластеров малого размера преобладающее влияние начинает оказывать поверхностная энергия, а октоэдрическое строение сильно проигрывает по этому показателю икосаэдрическим (Ih) или декаэдрическим (Dh) структурам, имеющим много меньшую площадь поверхности. Поэтому настоящая статья посвящена рассмотрению вопросов получения термически устойчивого икосаэдрического (декаэдрического) строения нанокластеров серебра диаметром до $2.0 \mathrm{~nm}$ у которых в качестве исходной фазы были приняты ГЦК или аморфная конфигурации, что позволяет проследить влияние начальной морфологии Ag-наночастиц на характер возможных структурных переходов с целью более глубокого понимания их природы.

Характерной особенностью атомов серебра является их относительно высокая реакционная способность, в результате чего для сохранения химически чистых $\mathrm{AgHЧ}$ необходимо использовать различные стабилизаторы, в частности лиганды. Другим возможным подходом к решению проблемы окисление/сульфидирования нанокластеров серебра может быть образование очень чистых частиц с идеальной кристаллической структурой [16], которые должны быть более устойчивыми к вышеупомянутым явлениям.

Мы считаем, что еще одной неучтенной возможностью повышения стабильности состава и формы АgНЧ может стать использование наночастиц серебра с размером, равным магическим числам различных структур. Данное предположение подтверждается экспериментальным анализом распределения синтезированных частиц по размерам (масс-спектром) который показал, что кластеры с определенным числом атомов, отвечающим магическим числам, гораздо более стабильны по сравнению с кластерами иной величины. При этом степень дефектности поверхности кластеров с „магическими“ числами атомов минимальна, в то время как у остальных она может быть весьма значительной.

В работах $[14,15]$ нами был проведен анализ влияния некоторых „магических“ чисел на устойчивость внутреннего строения малых AgHЧ, в том числе и с разным начальным расположением атомов [17]. Было показано, что характер термически индуцированных структурных переходов в исследуемых группах нанокластеров резко отличается, но полной стабилизации икосаэдрической

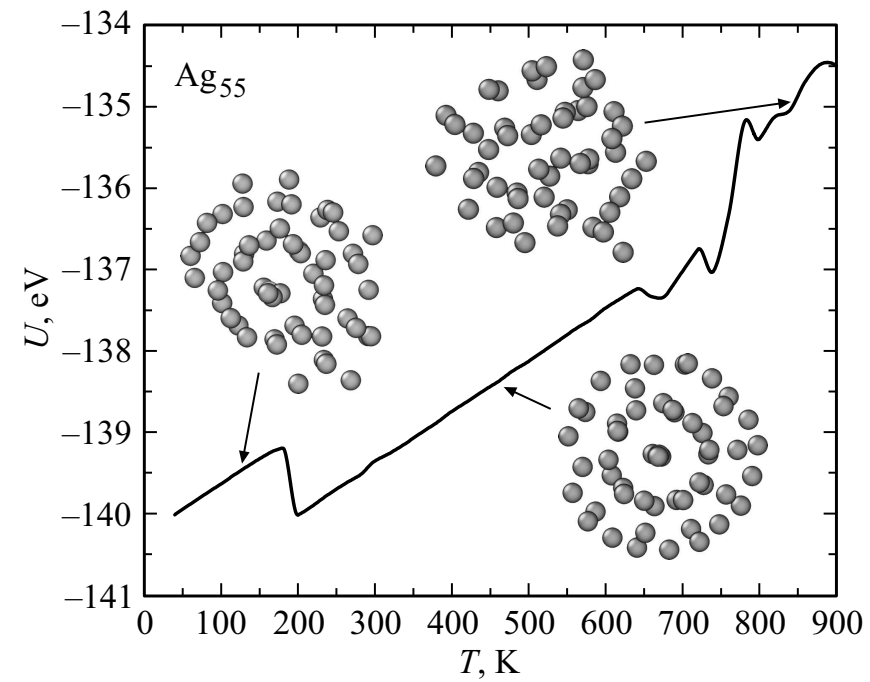

Рис. 1. Зависимость потенциальной энергии кластера $\mathrm{Ag}$ $(N=55$ атомов $)$ с начальным аморфным состоянием от температуры в случае наличия структурного перехода. Показан наиболее вероятный вариант термической эволюции.

(декаэдрической) фазы в ходе проведения компьютерных экспериментов все же зафиксировано небыло.

Поэтому в представленной работе мы сфокусируемся на исследовании влияния геометрических „магических чисел, соответствующих икосаэдрической симметрии, для чего рассмотрим нанокластеры серебра с числом атомов точно соответствующим геометрическим „магическим“ Ih-числам $\left(\mathrm{Ag}_{55}\right.$ и $\left.\mathrm{Ag}_{147}\right)$. Результаты проведенного моделирования показали, что при условии начального ГЦК-строения у самого малого по размеру кластера уже на предварительной стадии термической релаксации произошел политипный переход ГЦК $\rightarrow$ Ih и была сформирована идеальная икосаэдрическая структура. Никаких эффектов предплавления здесь не наблюдалось, зависимость потенциальной энергии теперь уже икосаэдрического кластера $\mathrm{Ag}_{55}$ от температуры имела строго линейный характер вплоть до температуры плавления, после чего происходило разрушение дальнего порядка.

В случае второго ансамбля частиц (с начальным аморфным состоянием) процесс термической эволюции строения претерпевал значительные изменения. Основными являлись уже два варианта термической эволюции, реализующиеся примерно в равной пропорции: первый - частицы $\mathrm{Ag}_{55}$ сохраняли начальное аморфное строение; второй - имел место структурный переход к Ih-модификации (рис. 1). Также из полученных данных видно, что потенциальная энергия нанокластера $\operatorname{Ag}_{55}$ с идеальным икосаэдрическим строением (первый ансамбль частиц) при $T=40 \mathrm{~K}$ составляла $-2.5761 \mathrm{eV} /$ atom, а в случае второго ансамбля частиц при сохранении аморфной субструктуры и переходе к Ih-строению она увеличивалась на $\Delta E=0.0272 \mathrm{eV} /$ atom и $\Delta E=0.0279 \mathrm{eV} /$ atom соответственно. Таким образом, 
рост удельной потенциальной энергии частиц $\mathrm{Ag}_{55}$ начальной аморфной морфологии, полученных в условиях экстремально быстрого охлаждения, всего лишь на $0.7 \mathrm{meV} /$ atom оказался способен существенно повлиять на характер дальнейшей термической эволюции кластера.

Попробуем объяснить полученный результат. В объемном состоянии серебро имеет гранецентрированную кубическую решетку, но в нанометровом диапазоне конкуренция между объемной и поверхностной энергиями может привести к формированию нескольких различных изомеров из-за очень близких значений энергий разных структур. Так МД-моделирование кластеров золота, проведенное в [18] с использованием потенциала Гупта, при $N=38$ атомов дает следующие величины свободной энергии кластера в своем основном состоянии: аморфном $(-3.4405 \mathrm{eV} /$ atom $)$, ГЦК ( $-3.44 \mathrm{eV} /$ atom $)$, икосаэдрическом $(-3.431 \mathrm{eV} / \mathrm{atom})$. Причем такое слабое различие у всех трех изомеров сохранялось вплоть до комнатной температуры. При $T>250 \mathrm{~K}$ тепловой энергии оказывается уже достаточно для преодоления потенциального барьера между разными структурами в результате чего случайным образом начинают встречаться все три изомера. В [19] также МД-моделированием были произведены аналогичные оценки для кластеров золота большего размера. Так, различие по энергии связи при $N=55$ составило $9.4 \mathrm{meV} /$ atom между аморфной и Ih-структурами, а при $N=75$ пятичастичная Dh-модификация оказалась стабильнее аморфной всего на $5.7 \mathrm{meV} /$ atom. То есть из-за близости энергий различных структурных типов даже небольшое ее изменение действительно может существенно поменять направление эволюции кластера.

Интересной особенностью замеченного нами структурного перехода ГЦК $\rightarrow$ Ih являлся его двухстепенчатый характер (рис. 1). То есть в ходе предварительной термической релаксации аморфного кластера $\mathrm{Ag}_{55}$ примерно в половине случаев начинался процесс перестройки внутреннего расположения атомов в сторону создания пятичастичной симметрии, что выливалось в формирование явно фиксируемого икосаэдрического зародыша. Однако в отличие от первой группы $\mathrm{Ag}_{55}$-кластеров (начальная ГЦК-фаза) икосаэдрическое строение здесь полностью все же не формируется и только с ростом тепловой энергии $(T \approx 200 \mathrm{~K})$ происходил окончательный переход к такому структурному типу.

Другим неожиданным моментом стало полное отсутствие структурного перехода в достаточно большом числе опытов для аморфного кластера $\mathrm{Ag}_{55}$. Мы считаем, что основной причиной стал тот факт, что аморфное строение характеризуется пониженным средним координационным числом по сравнению с ГЦК-расположением атомов, из-за чего в этом случае атомы имеют более высокую вероятность перемещения для формирования энергетически наиболее выгодного положения. Для рассматриваемых кластеров такого малого размера это может привести к минимально возможному значению поверхностной энергии из-за образования сферической формы, наблюдаемой нами при проведении компьютерных экспериментов. Однако ГЦК-кластер из-за особенностей своего строения (наличие больших плоских граней) таким значением поверхностной энергии обладать не может. Поэтому, если в ходе начальной термической релаксации в результате стохастических диффузионных процессов аморфный кластер попадает в состояние локального энергетического минимума, то может сложиться такая ситуация когда разупорядоченное строение может стать энергетически даже более выгодным, чем ГЦК. Этим же объясняется и двухступенчатый характер перехода ГЦК $\rightarrow \mathrm{Ih}$, фиксируемый нами в остальных случаях.

Таким образом, наличие начальной ГЦК-морфологии кластера $\mathrm{Ag}_{55}$ полностью гарантирует его переход в максимально возможную квазисферическую фасетную форму (Ih-строение), в то время как при начальном аморфном строении такая форма образовывалась только в 50-60\% проведенных опытов. В остальных случаях внешний вид кластеров $\operatorname{Ag}_{55}$ также был близок к сфере (без наличия фасет) при относительно низких температурах, но такое не плотно упакованное расположение атомов все же не может сохранить сферическую форму кластера при более высоких температурах в отличие от Ih-строения. Поэтому для случая кластера $\mathrm{Ag}_{55}$ начальная ГЦК-морфология может быть предпочтительней с точки зрения антибактериального действия $\mathrm{AgHЧ}$.

Второй рассматриваемый нами кластер $\left(\mathrm{Ag}_{147}\right)$ имел уже полностью заполненную третью Ih-оболочку, что для таких малых частиц может иметь решающее значение. Все это можно хорошо увидеть в случае проведенного нами анализа термического поведения кластера $\mathrm{Ag}_{147}$ с начальной ГЦК-фазой (рис. 2). Здесь, в отличие от $\mathrm{Ag}_{55}$, на предварительной стадии термической релаксации полного структурного перехода ГЦК $\rightarrow \mathrm{Ih}$ уже не наблюдалась, что говорит о малом выигрыше в поверхностной энергии при таком размере, из-за чего для преодоления энергетического барьера между политипными модификациями требуется уже большее значение тепловой энергии $k T$. В этом случае кластер $\mathrm{Ag}_{147}$ становится аналогом аморфного кластера $\mathrm{Ag}_{55}$ c Ih-преобразованием и для него также происходит двухступенчатый переход к пятичастичным структурам. На первом этапе до температур порядка 100-120 K наблюдается Ih-ядро, смещенное относительно центра частицы, с явно незавершенной внешней оболочкой кластера. Полученный результат подтверждается и работой [20], где было показано, что энергетическая стабильность кластера $\mathrm{Ag}_{147}$ понижается примерно на 30-35\% по сравнению с $\mathrm{Ag}_{55}$, становясь практически одинаковой с декаэдрическим кластером $\mathrm{Ag}_{146}$. Причиной данного явления является то, что в случае $\mathrm{Ag}_{55}$ примерно 76\% атомов лежат на поверхности кластера, в то время как у кластера $\mathrm{Ag}_{147}$ таких атомов только 62\%. Ясно, что изза меньшего координационного числа и, следовательно, 


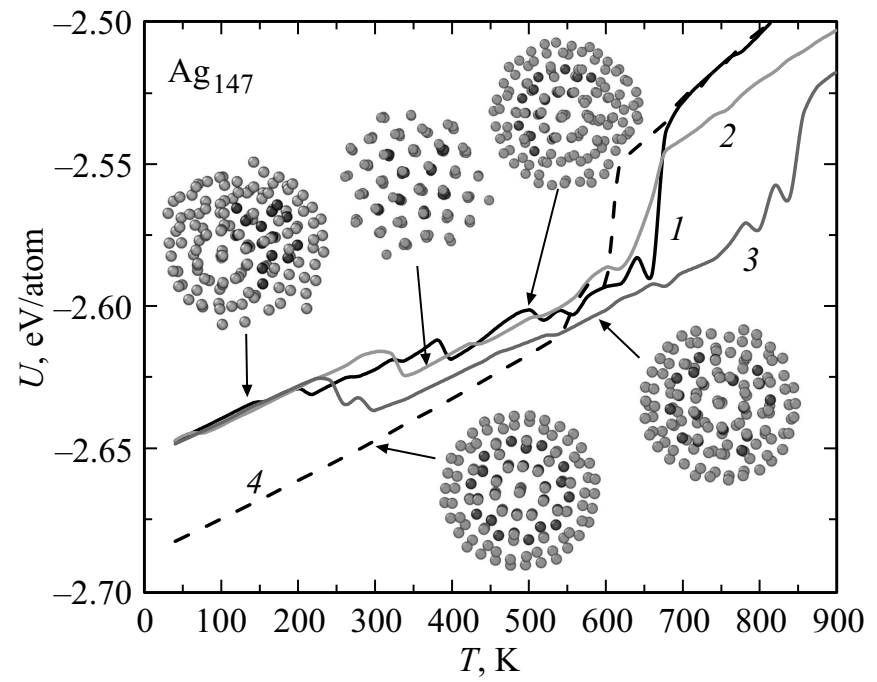

Рис. 2. Зависимость потенциальной энергии кластера $\mathrm{Ag}$ $(N=55$ атомов $)$ от температуры. Показаны наиболее вероятные варианты термической эволюции: 1 - начальное ГЦК-строение, 2 - начальное аморфное строение со структурным переходом к Dh-модификации, 3 - начальное аморфное строение со структурным переходом к Ih-модификации; 4 при охлаждении кластера из расплава. Для удобства оценки относительной стабильности строения энергия дана в eV/atom.

меньшего значения энергии связи, поверхностным атомам проще перемещаться по кластеру, формируя энергетически более выгодное построение. Именно поэтому первично ГЦК-кластер $\mathrm{Ag}_{147}$ сохранял свое искаженное Ih-строение до температур порядка $120 \mathrm{~K}$.

Далее происходила ожидаемая перестройка структуры, в результате которой наблюдались множественные ступенчатые изменения потенциальной энергии наночастицы, обусловленные оптимизацией формы и формированием Ih-кластера с уже завершенной внешней оболочкой $(70 \%$ опытов) или, в некоторых случаях (30\% опытов) декаэдра (Dh). Полное разрушение кристаллической структуры нанокластеров $\mathrm{Ag}_{147}$ происходило при разных температурах в зависимости от сценария термической эволюции. Таким образом, несмотря на то, что ГЦК-кластер $\mathrm{Ag}_{147}$ строго соответствовал геометрическому „магическому“ икосаэдрическому числу, в ходе нагрева могла быть сформирована как икосаэдрическая, так и декаэдрическая модификация, что указывает на близкие значения энергий данных пятичастичных структур [20].

Кривая 2 на рис. 2 соответствует случаю кластеpa $\operatorname{Ag}_{147}$ с начальным аморфным строением когда при подводе тепловой энергии происходил переход к Dh-модификации (20\% всех опытов) при температуре около $370 \mathrm{~K}$ с последующим ее разрушением при $T \approx 720 \mathrm{~K}$. Но наиболее интересной является кривая 3 , наблюдаемая для частиц второго ансамбля в подавляющем числе $(80 \%)$ опытов. Здесь кластер $\mathrm{Ag}_{147}$ с исходной аморфной морфологией формирует Ih-струк- туру в интервале температур от 300 до $400 \mathrm{~K}$. При этом внешняя оболочка имеет завершенную форму и $\mathrm{AgHЧ} \mathrm{остается} \mathrm{стабильной} \mathrm{вплоть} \mathrm{до} \mathrm{точки} \mathrm{плавления,}$ которая составляет порядка $840 \mathrm{~K}$. Следует отметить, что значения удельной потенциальной энергии $\mathrm{Ag}_{147}$ при $T=40 \mathrm{~K}$ во всех рассмотренных случаях фактически равны друг другу, а наибольший энергетический выигрыш при $T>300 \mathrm{~K}$ дает только переход из аморфного состояния в икосаэдрическое.

То есть, анализируя энергетическую составляющую стабильности строения наночастиц $\mathrm{Ag}_{147}$ с разным начальным строением (рис. 2) можно сделать достаточно четкий вывод, что именно такой переход обеспечивает наибольшую стабильность квазисферической фасетной формы в области комнатной температуры. Образование декаэдрического строения (кривая 2) ожидаемо приводит к чуть меньшей устойчивости, сопоставимой с устойчивостью икосаэдрического строения полученного при нагреве первичных ГЦК-кластеров $\mathrm{Ag}_{147}$.

В завершении было проведено сравнение первых трех вариантов термической эволюции со случаем медленного охлаждению $\operatorname{Ag}_{147}$ из расплава (кривая 4). Хорошо видно, что именно при такой методике частица формирует наиболее идеальное Ih-строение при температуре порядка $600 \mathrm{~K}$. Такая внутренняя структура отвечает наиболее устойчивой кристаллической конфигурации наночастицы $\mathrm{Ag}_{147}$ при $T<550 \mathrm{~K}$, а при $T=40 \mathrm{~K}$ кластер $\mathrm{Ag}_{147}$ обладает энергией $E=-7.1587 \mathrm{eV} /$ atom, что на $\Delta E=83 \mathrm{meV} /$ atom меньше случаев кривых $1-3 \mathrm{~K}$.

Следовательно, на основе проведенного компьютерного анализа процессов нагрева-кристаллизации (термоциклирование) наночастиц серебра с числом атомов соответствующим икосаэдрическим „магическим“ числам $\left(\mathrm{Ag}_{55}\right.$ и $\left.\mathrm{Ag}_{147}\right)$ и различным начальным строением (ГЦК и аморфное), а также полученных ранее результатов $[14,15,17]$ можно сделать вывод, что для образования устойчивого квазисферического строения с наличием большого количества фасет желательно использовать Ag-кластеры с „магическим“ Ih-размером. Кроме этого, для случая $\mathrm{Ag}_{55}$ лучшие результаты получаются при условии начального ГЦК-строения, а для кластера $\mathrm{Ag}_{147}$ наиболее стабильная требуемая форма может быть получена при процессе медленного охлаждения из расплава. Если не прибегать к данной процедуре, то независимо от начального строения в области комнатных температур все равно происходит формирование икосаэдрического или декаэдрического строения, однако уже не совсем идеального.

\section{4. Заключение}

В представленной работе при помощи компьютерного моделирования методом молекулярной динамики были исследованы вопросы термической стабильности внутреннего строения малых наночастиц серебра, 
соответствующих геометрическим „магическим“ числам икосаэдрической структуры с различной начальной морфологией. Было показано, что наличие начального ГЦК-строения кластера $\mathrm{Ag}_{55}$ полностью гарантирует его переход в максимально возможную квазисферическую фасетную форму, в то время как при начальном аморфном строении такая форма образовывалась только в 50-60\% проведенных опытов. В случае же кластеров большего размера требуемое строение было получено при любой начальной морфологии наночастиц серебра, однако в случае начального аморфного строения необходимый структурный переход происходил в области выше комнатной температуры, что является не совсем удобным с точки зрения практического применения. Таким образом, вид начальной конфигурации наночастицы определяет характер последующих термически индуцированных структурных переходов, а также в некоторых случаях влияет на стабильность формируемых кристаллических модификаций в области высоких температур. Поэтому был сделан вывод что с целью получения максимально устойчивого фасетного строения AgHЧ необходимо на предварительном этапе использовать процедуру термоциклирования.

\section{Благодарности}

Ю.Я. Гафнер благодарит научно-образовательный центр „Енисейская Сибирь“ за помощь в проведении исследований.

\section{Финансирование работы}

Работа выполнена при финансовой поддержке Российского фонда фундаментальных исследований (грант № 19-48-190002).

\section{Конфликт интересов}

Авторы заявляют, что у них нет конфликта интересов.

\section{Список литературы}

[1] P. Filipczak, K. Hałagan, J. Ulański, M. Kozanecki. Beilstein J. Nanotechnol. 12, 497 (2021).

[2] G. Kavei, A.M. Gheidari. J. Mater. Proc. Technol. 208, 514 (2008).

[3] G. Naz, H. Asghar, M. Ramzan, M. Arshad, R. Ahmed, M.B. Tahir, B.U. Haq, N. Baig, J. Jalil. Beilstein J. Nanotechnol. 12, 624 (2021).

[4] D.-P. Tran, H.-I. Lu, C.-K. Lin. Coatings 8, 212 (2018).

[5] S. Sharma, S. Shriwastava, S. Kumar, K. Bhatt, C.C. Tripathi. Opto-Electron. Rev. 26, 223 (2018).

[6] O. Urper, İ. Çakmak, N. Karatepe. Mater. Lett. 223, 210 (2018).

[7] Y. Ma, L. Zhi. Small Methods 3, 1800199 (2019).

[8] H. Sim, S. Bok, B. Kim, M. Kim, G.-H. Lim, S.M. Cho, B. Lim Angew. Chem. Int. Ed. 55, 11814 (2016).
[9] F. Pourcin, C.A. Reynaud, M. Carlberg, J.L. Rouzo, D. Duché, J.-J. Simon, L. Escoubas, R.-M. Sauvage, G. Berginc, O. Margeat, J. Ackermann. Langmuir 35, 2179 (2019).

[10] B.V. Ranishenka, A.Yu. Panarin, I.A. Chelnokova, S.N. Terekhov, P. Mojzes, V.V. Shmanai. Beilstein J. Nanotechnol. 12, 902 (2021).

[11] G.M. Misirli, K. Sridharan, S.M.P. Abrantes. Beilstein J. Nanotechnol. 12, 440 (2021).

[12] P. Filipczak, M. Pastorczak, T. Kardaś, M. Nejbauer, C. Radzewicz, M. Kozanecki. J. Phys. Chem. C 125, 1999 (2021).

[13] F. Cleri, V. Rosato. Phys. Rev. B 48, 1, 22 (1993).

[14] Л.В. Редель, Ю.Я. Гафнер, С.Л. Гафнер. ФТТ 57, 2061 (2015).

[15] Y. Gafner, S. Gafner, D. Bashkova. J. Nanopart. Res. 21, 243 (2019).

[16] S.M. Novikov, V.N. Popok, A.B. Evlyukhin, M. Hanif, P. Morgen, J. Fiutowski, J. Beermann, H.-G. Rubahn, S.I. Bozhevolnyi. Langmuir 33, 24, 6062 (2017).

[17] Д.А. Рыжкова, С.Л. Гафнер, Ю.Я. Гафнер. Письма в ЖЭТФ 113, 10, 668 (2021).

[18] I.L. Garzon, K. Michaelian, M.R. Beltan, A. Posada-Amarillas, P. Ordejon, E. Artacho, D. Sanchez-Portal, J.M. Soler. Eur. Phys. J. D 9, 211 (1999).

[19] I.L. Garzón, K. Michaelian, M.R. Beltrán, A. Posada-Amarillas, P. Ordejón, E. Artacho, D. Sánchez-Portal, J.M. Soler. Phys. Rev. Lett. 81, 1600 (1998).

[20] D. Liu, Z. Wen, Q. Jiang. Current Nanosci. 7, 463 (2011).

Редактор Т.Н. Василевская 